\title{
DINÁMICAINTERNA DEL GLOBO: INVESTIGACIONES SOBRE UNA NUEVA TEORÍA DE LA CAUSA DE LOS TEMBLORES
}

\author{
Luis Matamoros \\ San José, Costa Rica \\ Transcrito por: \\ Giovanni Peraldo Huertas \\ Escuela Centroamericana de Geología, Universidad de Costa Rica \\ gperaldo@geologia.ucr.ac.cr
}

\section{Tomado del documento original publicada por la Tipografía Nacional, San José, Costa Rica, 27 págs.}

\section{A Mr. De Lapparent \\ Dedica respetuosamente este el autor}

Fue después del terremoto de fines del año 1888 (29-30 de diciembre) cuando por primera vez nos ocupamos seriamente en el examen de los fenómenos de Dinámica Interna, tan comunes en estos países de la América Central.

En el año á que nos referimos, la altiplanicie central de Costa Rica fue azotada por una serie de temblores que terminó con el terremoto que acabamos de mencionar: recordamos perfectamente aún, que después del temblor de las once p.m. del 29 de diciembre y varios otros anteriores de menor intensidad, tuvimos bien presente en nuestra memoria las palabras de Mr. de Lapparent en su importante tratado de Geología, página 523: "On remarque que la plus forte secousse est rarement la première de la serie et qu`elle n`est jamais la dernière". Ello nos valió el haber estado preparados para salir de la habitación con la familia, al momento del terremoto que ocurrió á las 4 a.m. del día 30, ó sea, cinco horas después del más fuerte aviso que habíamos tenido.

Más tarde, cuando se presentaron otros movimientos de menor intensidad, pudimos averiguar, sin temor de equivocarnos, que el terremoto había pasado ya y que no había motivo para temer un temblor mayor.

Así se han confirmado aquellas palabras en todas nuestras observaciones posteriores, y después de diez años de estudio, manifestamos que la hipótesis de Mr. Lapparent es un verdadero principio que debía estar escrito con letras de oro en su monumental estudio.

La coincidencia de aquellos fenómenos seísmicos con otros importantes que se realizaban en el cielo en aquella misma época, y la comparación 
que desde 1888 hasta hoy hemos venido estableciendo en este sentido, no nos dejan ya lugar á dudas, y nos atrevemos á exponer una nueva hipótesis sobre la causa de los temblores, con la esperanza de que personas más competentes puedan ensancharla y completarla ó desvanecerla, con pruebas en contrario, y podemos advertir desde luego, que ninguna de las otras hipótesis admitidas satisface,á nuestro entender, tan bien los hechos que se observan, como lo que aquí exponemos.

\section{DIVISIÓN CARACTERÍSTICA DE LOS TEMBLORES}

Por razón de los efectos mecánicos de los temblores, pueden estos dividirse en dos clases perfectamente distintas: la primera es la que produce movimientos verticales, y en donde se nota que la mayor oscilación es en la parte superior de las casas, edificios, etc.; es el choque de abajo para arriba. La segunda constituye la de los movimientos horizontales ondulatorios, y casi siempre con manifestaciones de haber habido torsión, como si fuerzas apareadas accionaran en sentido inverso. La primera clase algunas veces con ligeras ondulaciones, se caracteriza por ser los temblores únicos y aislados; la segunda se manifiesta generalmente en series que siguen rigurosamente el principio de Mr. de Lapparent. Estudiaremos cada clase por separado.

\section{Clase}

Estos temblores, conocidos muy generalmente entre nosotros con el nombre de temblor de aire, pueden reconocer por causa la misma que atribuímos á los de la segunda, ó por lo menos á sus consecuencias; pero dadas las condiciones en que se verifican, bien pudiera pensarse que una dilatación ó contracción brusca de la corteza terrestre, bajo la influencia de la temperatura, los ocasiona.

Estos son los que algunos autores consideran que personas más o menos sensibles los presienten. En días extremadamente calurosos, tiempos de calma, y atmósfera por lo común muy despejada, ó con algunos stratus ó cirro-cú- mulos, inmóviles y perfectamente equilibrados, se asegura, con razón, que algún movimiento puede sobrevenir, y por lo regular acontece. Estos movimientos son de muy corta duración, afectan principalmente la parte superior de los edificios y altas construcciones y parece verdaderamente que el temblor viene del aire, pues casi es imperceptible en la superficie del suelo, mientras que se acentúa mucho más en los extremos superiores.

Siendo así ello sería una de las más preciosas pruebas que la Física del Globo nos da de que la masa terrestre es perfectamente elástica; pues en efecto, según las leyes del choque de los cuerpos si esta condición existe, ellos se comprimen en el momento del choque pero recobran su forma primitiva en seguida.

Sean dos masas m y m', homogéneas y dotadas de velocidades iniciales v y v', la una contra la otra: según la hipótesis de ser perfectamente elásticas, al momento del choque se comprimirán pero tomarán un instante después su forma primitiva. La masa $m$ habrá perdido una cantidad de velocidad $\mathrm{x}$, tal como si estuviere desprovista de elasticidad, y recibirá por consecuencia de la reacción otra velocidad igual á $\mathrm{x}$ en sentido inverso y tendrá, por tanto, una velocidad

$$
V-2
$$

La masa m' habrá adquirido una velocidad x' al momento del choque y otra velocidad x' por la reacción, y las velocidades de los dos cuerpos de masa m y m' serán

(1) $V=v-2 x$

(2) $V^{\prime}=v^{\prime}+2 x^{\prime}$

Como las velocidades adquiridas son inversamente proporcionales á las masas, podemos escribir

$$
\frac{x}{x^{\prime}}=\frac{m^{\prime}}{m} \quad x=\frac{m^{\prime} x^{\prime}}{m}
$$

Además, en el caso de choque de masas no elásticas, las ecuaciones generales son

$$
V=v-x=v^{\prime}+x^{\prime}
$$


luego

$$
\begin{aligned}
& \mathrm{x}=\frac{m^{\prime}\left(v-v^{\prime}\right)}{m+m^{\prime}} \\
& \mathrm{x}=\frac{m\left(v-v^{\prime}\right)}{m+m^{\prime}}
\end{aligned}
$$

y sustituyendo estos valores en la (1) y (2), nos da

$$
\begin{aligned}
& V=2-2 x=v-2 \frac{m^{\prime}\left(v-v^{\prime}\right)}{m+m^{\prime}}=\frac{2 m^{\prime} v^{\prime}+v\left(m+m^{\prime}\right)}{m+m^{\prime}} \\
& V^{\prime}=v^{\prime}+2 x^{\prime}=v^{\prime}+\frac{2 m\left(v-v^{\prime}\right)}{m+m^{\prime}}=\frac{2 m v+v^{\prime}\left(m^{\prime}-m\right)}{m+m^{\prime}}
\end{aligned}
$$

$\mathrm{Si}$ las masas son iguales, esto es $\mathrm{m}=\mathrm{m}$ ', las fórmulas (3) se convierten en

$$
\text { (4) } V=v-2 x=v^{\prime}
$$

$$
V^{\prime}=v^{\prime}+2 x^{\prime}=v
$$

Lo que quiere decir que los cuerpos cambiaron las velocidades y si v' $=0$, lo que indica que el cuerpo chocado estuviera en reposo al momento del choque,

$$
\begin{aligned}
& V=v-2 x=0 \\
& V^{\prime}=v^{\prime}+2 x=v
\end{aligned}
$$

Expresión importante que demuestra en el caso supuesto de v' = v que el cuerpo chocante quedó en reposo y transmitió toda la velocidad al cuerpo chocado.

Esto es precisamente lo que acontece en esa clase de temblores, y el aparato de Mariotte, que consiste en balas de marfil suspendidas y en contacto, procurando que sus centros queden en una línea recta, demuestra brillantemente aquella solución, pues que si se separa la primera bola dejarla caer chocando contra la segunda, ésta y las siguientes, sin salir de su estado de reposo, transmitirán el efecto á la última del choque, que saltará á su vez y caerá en seguida para hacer saltar de nuevo a la primera, etc., presentando así dicho aparato, al mismo tiempo, la idea clara y práctica del fenómeno que hemos analizado.

La predicción de estos movimientos seísmicos se hace casi imposible en el estado actual de la ciencia meteorológica, pues á nuestro entender, no son el resultado de un aspecto tranquilo que fácilmente se reconocería en el barómetro, sino el de una acumulación de hechos poco definidos y sometidos á influencias muy diversas. Los observatorios podrían indicarlos después de una larga serie de observaciones y estudios comparativos de las condiciones apuntadas anteriormente, no dejando de incluir también los motivos que creemos que determinan la $2^{\mathrm{a}}$ clase de temblores, porque se nota que en los lugares donde no existen volcanes estos movimientos tampoco aparecen sino alguna rarísima vez y esto como microseísmos perceptibles únicamente en los seismógrafos más delicados.

Debemos agregar que no se han observado nunca efectos desastrosos en esta clase de temblores. Su acción es instantánea, no se repiten con frecuencia, y pasado el temblor, generalmente el tiempo cambia, debido sin duda al desequilibrio producido en la atmósfera por la vibración.

\section{Clase}

La segunda clase de temblores, cuyos efectos y condiciones son muy distintos de los de la anterior, y que llamaremos desde ahora eléctricos, reconocen también causas diferentes.

Las teorías de Perrey, Quet y Falb, á mi modo de ver, se acercan mucho á la verdad sobre la causa de estos temblores, pero ellas no llenan muchas lagunas que quedan en la ciencia á este respecto y grandes objeciones se les pueden presentar. No es nuestro objeto hacer aquí la crítica de ninguna teoría ${ }^{1}$, sino presentar la nuestra, tal como la hemos concebido y estudiado, anotando desde luego que se acomoda á la explicación de todos los fenómenos que se presentan más que ninguna otra y muchísimo más todavía que las que han prevalecido en contra de las mismas que apoyamos.

La causa principal, única de estos temblores es la deformación de la masa del globo terrestre, bajo la influencia de los otros cuerpos celestes.

Sabido es que la figura teórica de una masa fluida, homogénea y sometida á las fuerzas de atracción newtoniana y centrífuga, es la de un elipsoide de revolución. 
En una memoria que elevámos á la academia, hoy Universidad de Laussanne, Suiza, y que fué coronada, presentámos una solución original y analítica de este problema, la cual, por lo corta, no tememos causar molestia al lector en exponerla.

Se trata, pues, de determinar la figura que tomará una masa fluida, homogénea y sometida á las fuerzas de atracción y repulsión considerando que ella gira sobre su eje.

Si se supone que la masa gire alrededor del eje de las Y, no tendríamos más que considerar las dos fuerzas $\mathrm{X}$ y $\mathrm{Z}$, y que la ecuación de equilibrio según la mecánica racional será

$$
\text { (1) } d p=\rho(X d x+Z d z)
$$

$\mathrm{X}$ y Z expresan la atracción de las moléculas del fluido y la fuerza centrífuga debida al movimiento de rotación, que consideramos como positiva, porque ella tiende á indicarnos que el cuerpo gira.

Si llamamos K el coeficiente newtoniano (número de Gauss), $r$ el radio vector y $\omega^{2}$ la fuerza centrífuga á la distancia unitaria, se tendrá, por la unidad de masa, las siguientes ecuaciones de las fuerzas

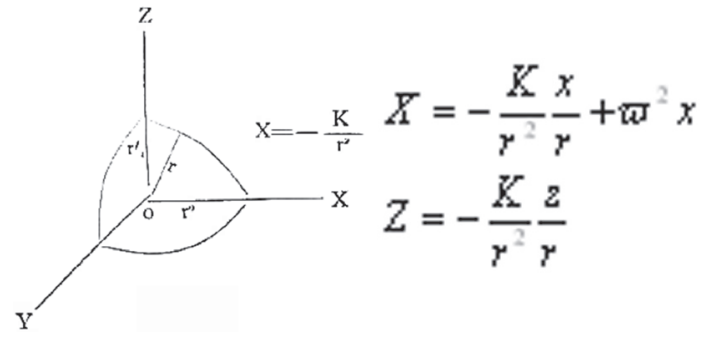

Si sustituimos estos valores en la ecuación (1), tendremos

$$
\begin{gathered}
\frac{d p}{\rho}=\left(-\frac{K}{r^{2}} \frac{x}{r}+\varpi^{2} x\right) d x-\frac{K}{r^{2}} \frac{z}{r} d z \\
\frac{d p}{\rho}=-\frac{K x d x+z d z}{r^{3}}+\varpi^{2} x d x
\end{gathered}
$$

pero como sabemos que $\mathrm{xdx}+\mathrm{zdz}=\mathrm{rdr}$, sustituyendo, nos dará

$$
\frac{d p}{\rho}=-\frac{K r d r}{r^{3}}+\omega^{2} x d x
$$

Integrando esta ecuación se obtendrá

$$
\frac{p}{\rho}=\frac{K}{r}+\frac{\varpi^{2} x^{2}}{2}+C
$$

donde admitimos $\rho$ como constante, por ser la masa fluida, homogénea, esto es, de densidad constante.

Para obtener la superficie libre es necesario también que la presión sea constante, esto es que

$$
\mathrm{dp}=0(3)
$$

lo que nos da

$$
\frac{K}{r}+\frac{\varpi^{2} x^{2}}{2}=C^{\prime}
$$

pero como por un valor de $\mathrm{x}=0$ se debe obtener $\mathrm{r}=\mathrm{r}^{0}$ la constante $\mathrm{C}^{\prime}$ queda determinada é igual á $\frac{K}{r^{0}}$

$$
C^{\prime}=\frac{K}{r^{0}}
$$

y sustituyendo su valor, resulta

$$
\frac{K}{r}+\frac{\omega^{2} x^{2}}{2}=\frac{K}{r^{0}}
$$

ó bien

$$
\frac{\varpi^{2} x^{2}}{2}=K \frac{r-r^{0}}{r^{0}}
$$


Pero como también por un valor de $\mathrm{x}=\mathrm{r}$ ', se obtiene que $r=r$, si reemplazamos estos valores en la ecuación anterior, se tendrá

$$
\frac{\Phi^{2} r^{2}}{2}=K \frac{r^{\prime}-r^{0}}{r r^{0}}(5)
$$

Si dividimos la ecuación (4) por la (5) obtendremos

$$
\frac{x}{r^{\prime 2}}=\frac{\frac{r-r^{0}}{r^{0}}}{\frac{r^{1}-r^{0}}{r^{\prime} r^{0}}}=\frac{\frac{r-r^{0}}{r}}{\frac{r^{1}-r^{0}}{r^{1}}}
$$
Habremos notado que la cantidad $\frac{r^{1}-r^{0}}{r^{1}}$
define el achatamiento del cuerpo girante.

Pongamos, pues, $\frac{r^{\prime}-r^{0}}{r^{\prime}}=\alpha$

y tendremos sustituyendo

$$
\frac{x^{2}}{r^{\prime 2}}=\frac{\frac{r-r^{0}}{r}}{\alpha}
$$

ó bien

$$
x^{2}=\frac{r^{2}}{\alpha}\left(I-\frac{r^{0}}{r}\right)
$$

Esta ecuación, ya de una forma bastante elegante, puede ser transformada en otra mejor y que permitirá resolver el problema propuesto

Si se extrae el valor $r$ de (6) se obtiene

$$
r=r^{0} \frac{r^{\prime 2}}{r^{\prime 2}-x^{2} \alpha}
$$

Reemplacemos $\mathrm{r}$ por su valor obtenido de la ecuación $r^{2}=x^{2}+z^{2}$ y se tendrá:

$$
\sqrt{x^{2}+z^{2}}=r^{0} \frac{r^{2}}{r^{2}-x^{2} \alpha}
$$

ó bien

$$
x^{2}+z^{2}=r_{0} r^{14} \frac{I}{\left(r^{2}-x^{2} \alpha\right)^{2}}
$$

que se puede escribir

$$
x^{2}+z^{2}=r^{02} r^{\prime^{14}}\left(r^{12}--x^{2} \alpha\right)^{-2}=r^{02} r^{14}\left(I-\frac{x^{2}}{r^{\prime 2}} \alpha\right)^{-2} \frac{I}{r^{14}}
$$

con el objeto de desarrollarla en serie según el binomio de Newton, lo que es posible en este caso porque $\mathrm{x}$ no será jamás mayor que $\mathrm{r}$, y por consiguiente $\frac{x}{r^{\prime}}$ será siempre menor que 1 .

El desarrollo nos da, eliminando las potencias superiores

$$
x^{2}+z^{2}=r^{02}\left(I+\frac{2 x^{2} \alpha}{r^{\prime 2}}\right)
$$

lo que es posible, porque siendo la cantidad $\mathrm{x} / \mathrm{r}^{`}$ ya muy pequeña, lo mismo que $\alpha$, es permitido en análisis despreciar todos los otros términos que contengan $\alpha^{2}$ en presencia de $\alpha$.

Tendremos así

$$
x^{2}\left(I-2 \alpha \frac{r_{0}^{2}}{r^{\prime 2}}\right)+z^{2}=r_{0}^{2}
$$

dividiendo por $\mathrm{r}_{0}^{2}$, resulta

$$
\begin{aligned}
& x^{2}\left(\frac{I}{r_{0}^{2}}-2 \alpha \frac{I}{r^{\prime 2}}\right)+\frac{z^{2}}{r_{0}^{2}}=I \\
& \text { pero como } \alpha=\frac{r^{\prime}-r_{0}}{r^{\prime}}, \text { el valor de } \mathrm{r}_{0} \mathrm{es} \\
& r_{0}=r^{\prime}(I-\alpha)
\end{aligned}
$$


y sustituyendo su valor en la anterior, se tendrá:

$$
x^{2}\left(\frac{I}{r^{\prime 2}(I-\alpha)^{2}}-2 \alpha \frac{I}{r^{\prime 2}}\right)+\frac{z^{2}}{r_{0}^{2}}=I
$$

Si desarrollamos otra vez en serie el valor de (1 - $\alpha)^{2}$ poniendo $r_{1}^{2}$ como divisor común, resultará

$$
\frac{x^{2}}{r^{\prime 2}}(I+2 \alpha-2 \alpha)+\frac{z^{2}}{r_{o}^{2}}=I
$$

lo que nos da

$$
\frac{x^{2}}{r^{\prime 2}}+\frac{z^{2}}{r_{0}^{2}}=I
$$

Pero esta ecuación muy conocida, es la de una elipse cuyos semiejes son $\mathrm{r}_{0}$ y $\mathrm{r}^{1}$

Y si esta elipse, á su vez, girando engendra lo que llamamos una elipsoide de revolución, y ella también indica la meridiana de la figura que se busca, luego, podemos concluir que si lo que investigamos es la figura de la tierra, considerándola como masa, fluida, homogénea, girando sobre su eje, y sometida a las fuerzas descritas, la figura de la tierra, decimos, es la de una elipsoide de revolución.

He aquí á lo que nos conduce el análisis más puro, más simple y más natural de la cuestión.

Debemos agregar que nuestra ecuación es general y no aplicable sólo á la tierra, sinó a la figura de todos los cuerpos celestes.

En un trabajo que preparamos, expondremos la teoría general para todos los cuerpos celestes, inclusive la figura del planeta Saturno, que entra también en nuestra fórmula fundamental.

Demostrando, como acabamos de hacerlo, que la figura de la tierra es la de un elipsoide de revolución, bajo las influencias combinadas de la atracción y de la fuerza centrífuga, natural es pensar que los astros vecinos influyen modificando, en razón de sus masas, distancias y posiciones relativas en el espacio, aquellas fuerzas, y por consiguiente deformen constante y continuamente el elipsoide. La teoría de las mareas confirma este resultado analítico.
Indudable es también que esta deformación produzca un frotamiento enorme de las moléculas que componen la masa, tanto mayor, cuanto mayores sean las masas atrayentes, hasta tomar la nueva forma de equilibrio que le corresponde, $y$ este frotamiento á su vez, desarrolle una gran cantidad de electricidad y calórico en proporciones hasta ahora desconocidas.

Esta gran masa eléctrica hará desarrollar, en lugares á propósito, una fuerte carga de inducción que en un momento dado descargue con energía muy superior á la de los rayos atmosféricos en otro punto de la masa hasta equilibrar su potencial.

Esa descarga eléctrica, á profundidades de la tierra mayores ó menores, es lo que constituye el temblor y sus consecuencias.

Bien puede suceder que sea una inducción eléctrica directa de los cuerpos celestes sobre la tierra, y que acompañado de calor producido por el frotamiento molecular desarrolle también una cantidad tan grande de éste, que ponga en ignición materias más o menos inflamables.

El hecho resulta también de que la chispa eléctrica, al atravesar masas de agua, debido á infiltraciones, descomponga estas en sus elementos, dando origen á grandes masas de gas combustible que á su vez se inflaman y produzcan las erupciones volcánicas.

$\mathrm{Si}$ hemos dicho anteriormente "en lugares a propósito" ha sido con marcada intención: indudable es que la deformación se produce y con ello el frotamiento y desarrollo de electricidad, pero ésta no induce sino en los lugares donde se hallan elementos capaces de cargarse, así como cuando un rayo atmosférico cae, no es sino la pila eléctrica del teléfono ó telégrafo la que recibe la carga inducida y hace vibrar el timbre ó instrumento de alarma.

Así, en nuestro caso, son sólo las masas volcánicas las que se cargan de electricidad. Esos centros, no son en nuestro concepto, sino unos grandes acumuladores hoy en descubierto, pero anteriormente focos de reunión de materiales inflamables, minerales descomponibles por la electricidad y los gases que se desarrollan, agua y otros elementos que los caracterizan.

Bajo la influencia de la electricidad y del calor, descompuestas unas materias, incandescentes 
otras, los vapores en presión han vencido las masas sólidas y escapado por grietas, lanzando hacia afuera materiales que interrumpen su paso.

En estas condiciones queda muy bien explicada la coincidencia de las erupciones volcánicas con los temblores; pero no es aceptable que el temblor de esta segunda clase sea consecuencia de la erupción; ésta más bien, es una consecuencia de aquél, y aquél, á su vez, es la consecuencia de la descarga eléctrica interna.

Bien pueden ocurrir ambos aisladamente, pues si la chispa eléctrica no atravesó líquidos que se descompusieran en vapores y gases que adquieran tensiones, solo ocurrió el temblor y no la erupción.

Se explica la concurrencia de ambos fenómenos, reconociendo al principio la misma causa, pero unos por descomposición de los líquidos como hemos dicho y otros por la descarga de la electricidad acumulada.

Cuando el terremoto de 1888, una comisión se encargó de examinar el volcán de Poás, de donde se creía provenían los movimientos seísmicos. La comisión declaró que una recrudescencia de actividad se notaba en el volcán extinto, cuyo cráter hoy solo tiene una laguna de agua con gran proporción de ácido sulfúrico diluido.

No es de extrañar, en efecto, según nuestra teoría, esta recrudescencia, pues muy posible fuera que la deformación del globo indujera en aquel punto una fuerte carga eléctrica descomponiendo las materias y poniendo en actividad la masa volcánica, al mismo tiempo que esa carga eléctrica se descargara, ya en la masa del volcán Irazú, ó en otro volcán cercano.

Por la teoría de la inducción eléctrica, que no creemos necesario repetir aquí, sabemos la influencia ejercida por un punto electrizado A sobre una esfera $\mathrm{C}$, aislada y primitivamente en estado neutro. El análisis demuestra, conforme á la experiencia de OEpinus, que la densidad eléctrica en un punto cualquiera $\mathrm{P}$, tiene por valor

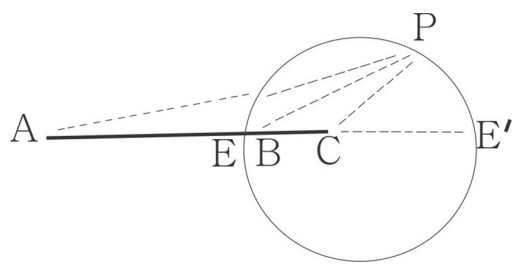

Revista Geológica de América Central, 37 Especial: 83-94, 2007 / ISSN: 0256-7024 en el punto E, que es el más cercano de la esfera al punto $\mathrm{A}$

$$
\mathrm{PA}=\mathrm{d}-\mathrm{R}
$$

y

$$
\rho E=\frac{m}{4 \pi} \frac{R-3 d}{d(d-R)^{2}}
$$

siendo $R<d$

Esta densidad es negativa y en el punto E', que es el más lejano, se tiene

$$
\rho E^{\prime}=\frac{m}{4 \pi} \frac{R+3 d}{d(d+R)^{2}}
$$

Su densidad es positiva y la línea neutra está determinada por la relación

$$
\frac{I}{d}-\frac{d^{2}-R^{2}}{P A^{3}}=O
$$

de donde

$$
P A=\sqrt[3]{d\left(d^{2}-R^{2}\right)}
$$

ecuación que representa un círculo cuyo plano es perpendicular á $\mathrm{AB}$, dividiendo la esfera, en dos zonas desiguales. La superficie de la zona negativa es más pequeña y tiende hacia cero cuando el punto de influencia se acerca indefinidamente; y tiende hacia la mitad de la esfera cuando el punto se aleja al infinito. Sería éste el caso de inducción producida directamente de los astros sobre el globo terrestre, y lo mismo podría hacerse respecto de un punto electrizado en el interior de la esfera, cuya densidad en función de la distancia $\mathrm{CB}=$ d`sería

$$
\rho=\frac{I}{4 \pi}\left(\frac{R^{2}-d^{1^{3}}}{R}\right) \frac{m}{B P^{3}}
$$

Es éste el valor que debe aplicarse en el caso de que tratamos, esto es, en el caso de inducción en las grandes masas volcánicas. 


\section{Ampliación de la teoría y comparación con los fenómenos observados}

Para ser verdaderamente ingenuos al exponer la teoría apuntada, repetimos que nuestro descubrimiento data desde 1888 , acentuando más á fines de ese año y corroborado con multitud de ejemplos y observaciones verificadas desde aquella época.

Testigos de ello han sido nuestro malogrado amigo el inolvidable Tessier, Ingeniero de la Dirección General de Obras Públicas, y varios otros distinguidos empleados de aquel centro, durante las ocasiones que he ocupado el puesto de Director é inspector del mismo.

Dio la casualidad, podría decir, que desde el año 1888, el "Bureau des longitudes" principió á publicar unas tablas, que contienen las épocas de los fenómenos celestes más notables del año: puede leerse en cualquiera de los prefacios de la "Connaissance des temps" publicados por aquella oficina, lo siguiente: "Depuis 1889, nous donnons sour le titre de Phénomènas, un tableau contenant les époques des phènomènes les plus importants del'annees".

Esas tablas fueron, por decirlo así, nuestra guía en un principio y cada vez que se presentaba un movimiento séismico de un carácter diferente al típico de los de primera clase, consultábamos las tablas de fenómenos, encontrando casi siempre una relación íntima entre el temblor y las posiciones de los cuerpos celestes más inmediatos á nuestro globo.

Hemos dicho que una inducción, ya sea directa de los cuerpos celestes, ya producida por las grandes masas electrizadas que provienen de la deformación de las moléculas del globo, motivada á su vez por mayores atracciones de los cuerpos celestes que exigen una nueva forma de equilibrio á nuestro globo, produce una cantidad de electricidad que se acumula en ciertos lugares que hemos designado ser especialmente los volcanes. Evidente es que siendo la tierra, no un conductor metálico, sino una masa discontínua, en donde la electricidad puede estar desigualmente distribuida, se pueden producir en el momento de la descarga una serie de chispas eléctricas que constituyan una longitud considerable, sobre todo lo cual se manifiesten los fenómenos seísmicos en el instante de la descarga, tal y como sucede en la atmósfera con los relámpagos que miden grandes longitudes y hacen vibrar á su aparición las capas de aire vecinos.

La teoría analítica de la figura de los relámpagos, muy ingeniosamente descrita por Mr. Paul Perrin (París, 1873), de la cual tenemos un precioso ejemplar en la Biblioteca Nacional, demuestra que la figura en zigzag es la que presenta menor resistencia al paso de la chispa ${ }^{2}$, y muy probablemente es que en el seno de la tierra, cuya densidad es mucho mayor que la del aire, esta figura sea más acentuada, y de ello resulta una de las pruebas más evidentes de nuestra teoría, como vamos a probarlo.

Los temblores de esta clase presentan casi siempre efectos de torsión en las columnas, y algunos movimientos caprichosamente aislados

Ninguna de las teorías hasta ahora emitidas satisface en la explicación de este fenómeno, mientras que la nuestra con sólo considerar la figura del relámpago eléctrico subterráneo, lo deje explicado.

Todavía nos quedan muestras en el cementerio de esta ciudad y en las columnas cuadradas del cuartel de Alajuela, que indican el fenómeno descrito de torsión acaecido con el terremoto de 1888 .

El tiempo de duración del temblor es otro de los motivos que nos hacen desechar la teoría de los gases y vapores. La duración del temblor en esta hipótesis no puede ser mayor que la producida por la expansión brusca de las masas en presión y debería tener siempre un máximum de intensidad al principio al ir en disminución hasta anularse mientras que observamos en la práctica que sucede todo lo contrario: los temblores largos principian casi insensiblemente, acentúan hacia el centro sus más grandes efectos de vibración, para disminuir después. Muchas veces el mismo temblor cesa repentinamente para aparecer un instante después con igual ó casi siempre con mayor intensidad. Se explica por el contrario fácilmente todo ello con nuestra teoría, admitiendo que la descarga eléctrica, verificada primero sobre una extensión corta y de gran resistencia, se ha disminuido ésta y se propaga enseguida con mayor intensidad y á mayores distancias, porque muchas veces la resistencia ya vencida, facilita una nueva descarga con menor 
potencial, por el mismo camino que atravesó la primera, tal y como sucede en la atmósfera, donde generalmente después de un relámpago sobreviene otro inmediato como si aprovechara el enrarecimiento ocasionado por el primero y utiliza el momento de mínima resistencia.

$\mathrm{Si}$, pues, como acabamos de comprobarlo, todos estos fenómenos de los temblores y los de los volcanes mismos, son la consecuencia de una grande electrización, y ésta á su vez, es ocasionada por la influencia de los cuerpos celestes, necesario es que alguna coincidencia los produzca en ciertas, y determinadas ocasiones.

Así lo es, en efecto, y desde los tiempos prehistóricos los antiguos turcos como un castigo divino la aparición de un eclipse, y no estuvieron nunca muy lejos de la verdad, como veremos enseguida.

\section{Efectos de los cuerpos celestes sobre la tierra}

Como acabamos de decirlo, los eclipses, así como las conjunciones de los astros con la tierra, han producido casi siempre grandes perturbaciones, y de aquí el honor de los antiguos al fenómeno extraordinario de un eclipse ó más bién al único fenómeno visible que se les presenta como causa de lo que sucedía en la tierra.

La ciencia nos dice hoy que no es el eclipse precisamente el que verifica la perturbación, sino la mayor masa de atracción ó inducción.

Las observaciones de Mr. Perrey en que sostiene que la luna en sus zizigías es el principal motivo de los temblores, y las de Falb que los atribuye á las mareas interiores producidas por la atracción de los astros, no están desprovistos de razón: son, en efecto, los astros los que con su mayor atracción y mayor masa inductiva, producen en la tierra los fenómenos que hemos descrito, y los huracanes, las tempestades, etc., pero de manera diferente, en mi concepto, de cómo lo proponen los eminentes sabios Perrey y Falb.

El Sol y la luna como más cercanos a la tierra, tienen, no hay duda, mayor influencia sobre ello, pero esta influencia se aumenta considerablemente con las masas de otros planetas que se interponen en ciertas ocasiones; así, cuando además de la conjunción de la luna se agregan las conjunciones de Mercurio y de Venus con ésta, sobre todo en los casos de menor distancia de la tierra, los fenómenos seísmicos aparecen; otras veces no son temblores sino huracanes, ciclones, etc., y cuando no éstos grandes lluvias, inundaciones y derrumbamientos, etc.

Algunas veces las conjunciones opuestas realizan un perfecto equilibrio en la masa terrestre y á pesar de su coincidencia no se presenta la menor perturbación, tal sucede cuando al mismo tiempo que se realizan las conjunciones de Mercurio y Venus con la luna y ésta con la tierra, se realizan las de Marte y Júpiter con la misma luna.

Un vacío nos queda por llenar, y este es el saber si podría determinarse el fenómeno que sobrevendría en una época dada, en que se realicen las consideraciones enteras.

Nosotros lo dudamos. La teoría precedentemente expuesta sobre la influencia de los cuerpos electrizados (los astros) sobre una esfera (la tierra) en estado neutro, ayudará poderosamente á esa investigación. Si además se determina astronómicamente el punto ó puntos principales afectados de la esfera terrestre, y la línea, eje central de todos los cuerpos en conjunción simultánea, es muy probable que la posición de esta línea con respecto á los lugares más o menos aptos para ser influenciados, produzcan unos ú otros efectos. Nada de extraño tendrá que si el punto ó puntos afectados fueran en los centros de los océanos, entonces no se produjeran más que ciclones y grandes tempestades, y si estos puntos fueran cerca de los volcanes, su producto sería temblores, sacudimientos y erupciones.

No nos alarma, pues, que la estadística confirme que los más fuertes temblores sobrevienen en los perigeos de la luna, cuando la tierra está más cerca del sol.

Evidente es que el medio en que nos encontramos nos priva del placer de extender más allá nuestras observaciones y cálculos. Dejamos á los sabios este importante trabajo que nuestros recursos no nos permiten proseguir, pero si creemos y estamos seguros de que, determinando exactamente la posición astronómica de los cuerpos celestes más cercanos, en los momentos de los fenómenos seísmicos ya observados é inscritos en las cronologías especiales, puede obtenerse una ley para prever los temblores futuros y también 
para todas las otras perturbaciones geológicas y atmosféricas ya indicadas.

Los trabajos de Mr. de Montessus en la República del Salvador, serán de grande importancia en la persecución de esta admirable ley, y determinada que sea, lo repetimos, no se nos culpe de incompetencia en perseguirla, porque creemos que hemos sentado sus bases, sino de falta de medios y recursos para comprobarla.

La solución de este importante desideratum de nuestra teoría es, en efecto, relativamente sencillo. Si examinamos los fenómenos seísmicos desde el punto de vista de las leyes del malogrado Mr. Quet, sobre la inducción solar muy probable es que todo se origine de esta inducción, importante si á la masa del sol se agregan las otras masas de los planetas intermedios entre el Sol y la tierra incluso nuestro satélite, la luna.

Consiste, pues, en una simple comparación entre elementos en que se verifica la inducción, el lugar donde se verifica y el momento y lugar donde se verifica el temblor o la perturbación terrestre.

Sentimos mucho no tener á nuestro alcance la teoría de Mr. Quet.

\section{Prevención de los daños}

Demostrada fundamentalmente la causa de los temblores y erupciones; establecida como acabamos de hacerlo, que las erupciones son una consecuencia de los temblores, es decir, el resultado de la descarga eléctrica subterránea á través de masas líquidas que se han volatizado y descompuesto en gases inflamables procede investigar si sería fácil o nó prevenir los daños y aún evitar los mismos temblores (a).

Establecida la causa, fácilmente se obtiene su remedio. Conocido el diagnóstico, procede la cura.

Nosotros creemos que ese procedimiento es muy sencillo y poco costoso. Puesto que todo proviene de la acumulación de grandes masas de electricidad, lo que se necesita es neutralizarla estableciendo corriente, y en este caso bastaría con introducir en los cráteres de los volcanes en actividad un cable que transporte la electricidad allí acumulada á los océanos ó grandes masas de aguas. Es, en suma, el establecimiento de pararrayos (b) en cada volcán para transportar a la atmósfera ó á las aguas esos grandes excesos de electricidad acumulada, con positivo éxito para la tranquilidad de los lugares amenazados.

$\mathrm{Si}$ esa electricidad puede usarse alguna vez industrialmente, sería ello la mejor comprobación de la teoría que exponemos. Ya utilizamos las mareas, el resultado físico de la deformación; utilicemos ahora el calor y la electricidad, resultados físicos del frotamiento por la deformación.

Ningún comentario se necesita después de leída esta teoría para confirmar la causa de la destrucción de S. Pierre ${ }^{3}$.

Las causas alegadas en el "Scientific American", Junio de 1902, cuya traducción publicó "El Derecho" en sus números 195 y 197 de 12 y 14 de Junio próximo pasado, no satisfacen en nada á los fenómenos ocurridos, así como tampoco lo que publicó "La Nature" de París en su número 1513.

Siendo estos documentos extremadamente novedosos, nos corresponde, pues, involucrar nuestro exclusivo derecho de propiedad intelectual del artículo preincerto.

\section{Calor interno del Globo}

De la teoría precedente, se deduce lo observado de la hipótesis que ha prevalecido hasta hace algunos años sobre el fuego central. Desmentida desde luego y entre otras causas por la simple determinación de la densidad del globo $(5,5)$ cuando el término medio de la densidad de todo el cuerpo de su superficie no excede de 3 , viene a destruirse totalmente con lo nuestro. En nuestro concepto la deformación es la que causa el calor; este calor será tanto mayor cuanto mayor sea la distancia al eje de la tierra. En la zona ecuatorial esa distancia es máxima, hasta su superficie, más conforme se aproxima al eje central la temperatura debe disminuir en razón de que las moléculas tendrán menor deformación cuanto más cerca se encuentran de ese eje; que es inmóvil. En nuestro sentir, la temperatura del centro debe ser igual á la temperatura interplanetaria esto es de $273^{\circ}$ bajo cero centígrado, ó sea el cero absoluto de la temperatura que se traduce por una vibración nula de las moléculas.

Manifestada esta consecuencia de la teoría, á nuestro ilustrado amigo don Juan Fernández Ferraz (c), nos llamó la atención de consignar el 
hecho resultado de ello las zonas en que se divide el globo, quedando establecida la zona tórrida para las partes más lejanas del eje, de muy vibración de sus moléculas, la zona templada más cercana al centro y por último la zona fría de los polos en donde la deformación es nula. No se concibe de otro modo, dice el señor Ferraz, que aquellos parajes bañados por el sol durante 6 meses al año están constantemente fríos.

San José, 12 de julio de 1900

Luis Matamoros

Ingeniero Civil

\section{Notitas al pie}

(a) Aplaudiendo sinceramente el trabajo científico de mi buen amigo el Ingeniero don Luis Matamoros, quien en efecto comenzó sus observaciones sobre la materia en la fecha que citó, estando ambos con nuestras familias acampados en el patio de la casa de doña Inés Ugalde v. de Iglesias, de Alajuela, mientras yo leía el interesante estudio a los volcanes y terremotos de Zurcher y Marcollé, pienso que mi amigo debió hacer algún viaje, antes de dar a luz su teoría, a fin de contrastarla y perfeccionarla, en un observatorio astronómico y meteorológico magnético. De toda suerte le creo sobre la pista de un gran descubrimiento.

(b) Antipararrayos llamé yo en esos días el aparato que ideaba capaz de descargar en la atmósfera la electricidad desarrollada por la acción físico - química interior. Mi imaginación, en presencia de las grietas superficiales producidas por el temblor en derredor del Poás, veía ese fenómeno como el rompimiento de un vidrio, por una pedrada, y de allí que escribiera á mi malogrado amigo don Pío Víquez, entonces director del diario La República, que me parecía que los efectos desastrosos de los temblores se propagan por la superficie, como toda corriente eléctrica inducida y que así con habitaciones subterráneas á pocos metros de profundidad podríamos librarnos de ellos, y aún más, lucubraba yo, se podrían descargar á la atmósfera directamente esa electricidad acumulada con una especie de pararrayos invertido ó antipararrayos. El descubrimiento de
Figers de Canarias, de que últimamente ha dado del cable; de un aparato para el aprovechamiento directo de la electricidad atmosférica, me hace ahora sospechar que todos vamos en camino de algo muy interesante. El señor Matamoros, que acaba de irse á los Estados Unidos, nos dirá pronto algo á este respecto.

(c) Demaciado bondadoso fué el señor Matamoros en hacer constancia de mis observaciones acerca de su de su importante trabajo. Por ello le debo gratitud. Lo indicado por mí con respecto á la temperatura del globo terrestre, - y que naturalmente se aplica á cualquier otro cuerpo celeste, - fué que el frío de los polos nos daba a entender que todos los puntos del eje que los une - en igual reposo relativo - debe tener igual temperatura, advirtiéndose por la inclinación y declinación de los polos y por la precesión de los equinoccios que vendrían a formar como dos conos opuestos por los vértices, coincidiendo el punto de contacto de éste con el centro de figura del globo y siendo sus bases dos planos ideales tangentes á los polos, de donde formaron de zonas frías, templadas y tórridas.

No doy á este pensamiento más valor que el de una bella idea, y pienso que el señor Matamoros ha exagerado asignándolo como importante

San José, 28 de julio de 1902

Juan F. Ferráz

\section{Notas}

${ }^{1}$ El señor de Lapparent da un resumen de éstas en la página 542: "Influence des phenomènes astronomiques" pero según lo que se va á exponer, se comprenderá que la nuestra está fundada sobre una base muy diferente.

${ }^{2}$ Lógicamente esa figura en zigzag tiene su explicación. En efecto, una vez lanzada una chispa en cierta dirección rectilínea dada, ha tenido en su trayecto una gran resistencia y ha descompuesto las moléculas del fluido que ha atravesado, rarificando su densidad en los espacios vecinos. En un momento dado la resistencia ha crecido extraordinariamente hasta el punto de que la chispa, obedeciendo al principio de mínima acción, encuentra mayor facilidad de retroceder 
para caminar por un espacio menos resistente; en cierto límite hay retroceso otra vez, por el mismo motivo y de ahí la forma típica del zigzag.

${ }^{2}$ El rayo eléctrico subterráneo, al lanzarse en cierta dirección, arrastrará un cuerpo libre en ese mismo sentido; pero con motivo de la figura del camino que debe recorrer, este cuerpo libre será casi instantáneamente impulsado por otra fuerza igual á la primera y con dirección casi contraria de donde resulta que el cuerpo libre fue sometido á un par de fuerzas que produjo la rotación.

${ }^{3}$ El desastre de Martinica viene á confirmar estas teorías. Los primeros peligros se notaron el 3 de mayo. El 8 de mayo á las 7:50 a.m. tuvo lugar la erupción que acabó en segundos con 28,000 habitantes. El 7 de mayo ocurrió el eclipse parcial de sol $(0,858)$ y el 8 á las $5^{\text {h }}$ Mercurio estaba en conjunción con la luna. La mayor fase tuvo lugar á las 10.43.8 t.m. de París, en el lugar cuya longitud fue de $126^{\circ} 49^{\prime}$ Oeste, y latitud $70^{\circ} 18$ Sur. Los fenómenos aparecen en Montagne Pelée, situada á 1351 metros por una longitud de $63^{\circ} 30^{\prime} 22^{\prime \prime}\left(4^{\mathrm{h}} 13^{\mathrm{m}} 38^{\mathrm{s}} 9\right)$ Oeste y $14^{\circ} 48^{\prime} 52$ N. ¿Qué relación existe entre las fechas astronómicas y la situación de los lugares? No podemos decirlo, pero sí establecemos que esos grandes fenómenos ocurridos en las Antillas menores han venido corroborar nuestra teoría que ahora nos atrevemos á publicar sin vacilar. 